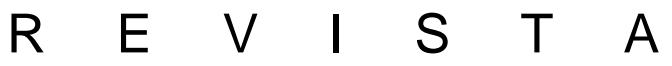

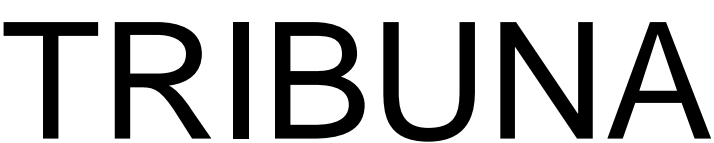 \\ INTERNACIONAL \\ Publicación del Departamento de Derecho Internacional \\ Volumen 3 / N5 / 2014
}

FACULTAD DE

DERECHO

UNIVERSIDAD DE CHILE 


\section{Rector de la Universidad de Chile}

Víctor Pérez Vera

Av. Alameda Libertador Bernardo O’Higgins 1058, Santiago

\section{Representante legal}

Roberto Nahum Anuch

Decano de la Facultad de Derecho de la Universidad de Chile

\section{Director Responsable}

Mario Ramírez Necochea

\section{Editora de Contenidos}

Rita Lages

\section{Comité Editorial}

Íñigo Álvarez Gálvez (Universidad de Chile, Chile) Gonzalo Aguilar (Universidad Andrés Bello, Chile) José Carlos Fernández Rosas (Universidad Complutense de Madrid, España)

Claudio Grossman (American University, EE.UU) Mattias Kumm (New York University, EE.UU) Hugo Llanos (Universidad Central, Chile) Cecilia Medina (Universidad Diego Portales, Chile) Elina Mereminskaya (Universidad de Chile, Chile) Mónica Pinto (Universidad de Buenos Aires, Argentina)

\section{Revista Tribuna Internacional M.R.}

Publicación del Departamento de Derecho Internacional de la Facultad de Derecho de la Universidad de Chile. Su objetivo es fomentar la reflexión, el debate, el análisis y la comunicación sobre el derecho internacional en forma pluralista y con rigor científico. Se publica cada semestre en los meses de junio y diciembre mediante convocatoria abierta a la publicación de artículos y monografías inéditos, comentarios de jurisprudencia, recensiones y comentarios de libros, en los campos de derecho internacional, derechos humanos y relaciones internacionales, tanto en castellano como inglés.

Volumen 3/ N 5 / 2014

www.tribunainternacional.uchile.cl

ISSN 0719-210X (versión impresa)

ISSN 0719-482X (versión en línea)

Departamento de Derecho Internacional

Facultad de Derecho

Universidad de Chile

Av. Santa María 076, $4^{\circ}$ piso

Providencia, Santiago de Chile

\section{Diseño y producción:}

Gráfica LOM

www.lom.cl

Impreso en Chile/ Printed in Chile

Se autoriza la reproducción total o parcial del contenido de la publicación, siempre que se reconozca y cite el/ la/ los/ las autor/a/es/as y la publicación, no se realicen modificaciones a la obra y no se la utilice para fines comerciales. 


\title{
Reflexiones sobre el derecho internacional y la delimitación marítima. Comentario del fallo de la Corte Internacional de Justicia en el caso Perú c. Chile
}

Reflections on international law and maritime delimitation. A commentary on the judgment Peru $v$. Chile of the International Court of Justice

\begin{abstract}
Francisca Aguayo
aguayo.francisca@gmail.com

Licenciada en Derecho, mención en Derecho Internacional y Europeo. Master en Derecho Internacional y Organizaciones Internacionales, Universidad Paris 1 Panthéon-Sorbonne. Candidata a Doctor en Derecho Internacional Público y asistente doctoral en Derecho Internacional Económico y Relaciones Internacionales, Universidad Paris 1 Panthéon-Sorbonne. Ex becaria de la Academia de Derecho Internacional de La Haya, sesión de Derecho Internacional Público.
\end{abstract}

Resumen: El fallo de la Corte Internacional de Justicia del 27 de enero de 2014 relativo a la delimitación marítima entre Perú y Chile reconoció que una frontera marítima a lo largo del paralelo geográfico había sido convenida de manera tácita por las Partes hasta las 80 millas marinas. Desde esa distancia hasta las 200 millas marinas, la Corte estableció una frontera de novo, siguiendo el método de la equidistancia conforme a la solución subsidiaria al acuerdo de los Estados prevista por el derecho marítimo internacional, tal y como ha sido interpretada por la jurisprudencia de la Corte. El fallo reconoció, además, el punto en que llega al mar el paralelo que pasa por el hito n. ${ }^{\circ} 1$ como punto inicial de la frontera convenida. Cinco declaraciones individuales, cinco opiniones disidentes -una individual y otra común de cuatro jueces- y dos opiniones individuales fueron anexadas al fallo. La proliferación de posiciones individuales pone en evidencia dificultades irreconciliables en el razonamiento de la Corte, que parecen indicar la adopción de una solución de compromiso sin sustento jurídico sólido.

Palabras clave: frontera marítima, acuerdo tácito, solución subsidiaria, proliferación de opiniones individuales, solución de compromiso.

Abstract: The judgment pronounced on the 27th of January 2014 by the International Court of Justice on the maritime dispute between Chile and Peru recognized the existence of a maritime boundary passing through the geographical parallel, which had been set by a tacit agreement between the Parties until 80 nautical miles. From that point until 200 nautical miles, the Court established a boundary de novo, by using the equidistance method in 
accordance with the subsidiary solution prescribed by International Maritime Law as the Court has interpreted it. Moreover, the judgment recognized the starting-point of the agreed boundary as the intersection of the geographical parallel passing through Boundary Marker $n^{\circ} 1$ with the low-water line. Five declarations, five dissenting opinions one individual and another common to four judges - and two separate opinions were enclosed to the judgment. The proliferation of individual positions makes evident the existence of irreconcilable differences in the Court's reasoning, which seem to indicate the adoption of a compromise solution without solid legal bases.

Key words: maritime boundary, tacit agreement, subsidiary solution, proliferation of individual opinions, compromise solution.

\section{Introducción}

El diferendo marítimo entre Chile y Perú remonta oficialmente a principios de los años 2000, cuando Perú comunicó a Chile, el 20 de octubre de 2000, y al Secretario general de las Naciones Unidas, el 9 de julio de 2001, su desacuerdo respecto del hecho que Chile considerara el paralelo que pasa por el hito n. 1 como frontera marítima entre las Partes. El 19 de julio de 2004, Perú propuso formalmente a Chile iniciar negociaciones al respecto, que fueron rechazadas por éste último el 10 de septiembre del mismo año.

El 16 de enero de 2008 Perú presentó la demanda contra Chile frente a la Corte Internacional de Justicia -en adelante "CIJ"-, sobre la base del Tratado americano de soluciones pacíficas - "Pacto de Bogotá" - adoptado en el marco de la Organización de los Estados Americanos el 30 de abril de 1948. En efecto, la competencia de la CIJ, así como de toda jurisdicción internacional, debe fundarse en el consentimiento de las Partes. En el caso de la CIJ, el consentimiento puede establecerse por medio de la declaración de aceptación facultativa de la jurisdicción obligatoria de la Corte, prevista por el artículo 36, parágrafo 2, de su Estatuto. El artículo XXXI del Pacto de Bogotá estipula que conforme a dicha disposición "las Altas Partes Contratantes declaran que reconocen respecto a cualquier otro Estado Americano como obligatoria i convenio especial mientras esté vigente el presente Tratado, la jurisdicción de la expresada Corte en todas las controversias de orden jurídico que surjan entre ellas y que versen sobre [...] b) Cualquier cuestión de Derecho Internacional". Por este motivo, y pese a que la interposición de excepciones preliminares relativas a la incompetencia de la CIJ fue considerada en su momento por Chile, la competencia de la Corte no fue finalmente cuestionada y el debate se concentró directamente en las cuestiones de fondo. 
En su demanda, Perú solicitaba a la Corte que estableciese la frontera marítima con Chile de novo, dado a que ésta, según Perú, nunca había sido convenida por ambos Estados. Sobre la base del derecho internacional general y la jurisprudencia de la CIJ, Perú solicitaba el trazado de una línea equidistante desde el Punto de la Concordia, donde comenzaba, según Perú, tanto la frontera terrestre -conforme al Tratado de Lima de 1929- como la frontera marítima. En su respuesta, Chile pedía a la Corte rechazar la demanda peruana, invocando varios instrumentos jurídicos, especialmente la Declaración de Santiago de 1952, que establecían y confirmaban la frontera marítima entre las Partes. Esta frontera, según Chile, seguía el paralelo geográfico que atravesaba el hito n. ${ }^{\circ}$ 1, punto que había materializado el inicio de la frontera terrestre y que servía entonces, según Chile, como punto inicial de la frontera marítima.

El estudio rápido de las normas de derecho internacional general aplicables al caso (II) es necesario para analizar el razonamiento de la Corte, tanto respecto de la existencia de la frontera y su extensión (III), como de su vocación y punto inicial (IV). Las dificultades así observadas permitirán formular una conclusión sobre la solución adoptada por la Corte (V).

\section{Derecho internacional general aplicable}

Las normas de derecho internacional determinantes en el caso fueron las normas consuetudinarias de derecho marítimo, tal y como han sido interpretadas por la CIJ, las normas internacionales de interpretación de tratados y la jurisprudencia de la CIJ relativa a la existencia de un acuerdo tácito en materia de fronteras marítimas.

El principal instrumento internacional relativo al derecho marítimo es actualmente la Convención de las Naciones Unidas sobre el Derecho del Mar (CONVEMAR), adoptada el 10 de diciembre de 1982. De las dos Partes, sólo Chile ha firmado y ratificado la CONVEMAR $^{1}$, por lo que, en virtud del principio del efecto relativo de los tratados ${ }^{2}$, la CONVEMAR no podía ser aplicada para resolver el diferendo. No obstante, las reglas contenidas en la Convención relativas a los espacios marítimos y a su delimitación son consideradas como el reflejo del estado actual del derecho internacional consuetudinario ${ }^{3}$. Con respecto a los espacios marítimos, la CONVEMAR cristalizó la formación de una norma consuetudinaria relativa a la existencia de derechos marítimos del Estado costero hasta las 200 millas marinas -bajo la apelación de "zona económica exclusiva" (ZEE)-,

1 Ratificada el 25 de agosto de 1997 y promulgada por Decreto No 1.393 el 28 de agosto de 1997.

2 Norma consuetudinaria codificada en el artículo 34 de la Convención de Viena sobre el Derecho de los Tratados. Véase fallo, párr. 57. Todas las traducciones del fallo son propias de la autora. 
norma que no existía en el marco de las convenciones de Ginebra de 1958 que regulaban hasta ese periodo el derecho marítimo internacional. Asimismo, los artículos 15, 74 y 83 relativos a la delimitación entre Estados con costas adyacentes del mar territorial, de la ZEE y de la plataforma continental reflejan normas consuetudinarias. La lógica contenida en estas normas para efectuar la delimitación es la misma: el acuerdo entre las Partes prima (1) y, sólo en su defecto, se aplican soluciones subsidiarias para efectuar la delimitación (2).

\section{La prioridad del acuerdo entre las Partes y la importancia de las normas de interpretación de tratados}

Para pronunciarse sobre la existencia de un acuerdo internacional entre las Partes, es esencial proceder a la interpretación de los instrumentos jurídicos pertinentes para la resolución del caso. Para esto, la Corte aplica las normas consuetudinarias de interpretación de tratados, codificadas en los artículos 31 y 32 de la Convención de Viena sobre el Derecho de los Tratados adoptada el 23 de mayo de 1969, a la que tanto Chile como Perú son Partes". De manera general, el artículo 31 establece que "[u]n tratado deberá interpretarse de buena fe conforme al sentido corriente que haya de atribuirse a los términos del tratado en el contexto de estos y teniendo en cuenta su objeto y fin". El artículo 32 prevé por su parte la posibilidad de recurrir a "[m]étodos de interpretación complementarios" -principalmente los trabajos preparatorios y las circunstancias de celebración del tratado- para confirmar o establecer el sentido del texto si éste permanece ambiguo o conduce a un resultado manifiestamente absurdo.

Si el sentido del texto no permite establecer un acuerdo entre las Partes, entonces, el acuerdo sobre la delimitación marítima -que excluirá la aplicación de la solución subsidiaria- podrá todavía ser tácito. En efecto, el derecho internacional no es formalista, por lo que no exige una determinada forma, ni apelación, para la existencia de un acuerdo internacional vinculante. Lo fundamental es establecer el consentimiento de las Partes respecto de una determinada norma. Sin embargo, siendo las fronteras marítimas un tema altamente importante para los Estados, la CIJ ha precisado que un acuerdo tácito en esta materia "no debe ser presumido fácilmente"s.

4 Chile ratificó la Convención el 9 de abril de 1981 (promulgada por el Decreto $\mathrm{N}^{\mathrm{o}} 381$ del Ministerio de Relaciones Exteriores) y Perú el 14 de septiembre del 2000 (Decreto Supremo Nº 029-2000-RE).

5 CIJ. Controversia territorial y maritima entre Nicaragua y Honduras en el mar del Caribe (Nicaragua contra Honduras), fallo del 8 de octubre de 2007, párr. 253. 


\section{A defecto de acuerdo, la aplicación de la regla subsidiaria}

A defecto de un acuerdo -expreso o tácito- que establezca la frontera marítima, se utiliza la regla subsidiaria prevista por el derecho internacional consuetudinario, tal y como se encuentra reflejado en la CONVEMAR. Esta solución varía según el espacio marítimo en cuestión: en el mar territorial, se trata de la regla de la línea equidistante (artículo 15), mientras que en la ZEE y en la plataforma continental, las normas internacionales solo prescriben la necesidad de encontrar una solución sobre la base de la equidad (artículos 76 y 83).

En su práctica reciente, la CIJ ha utilizado de manera relativamente constante un método en tres etapas para establecer la frontera sobre la base de la equidad en la ZEE y en la plataforma continental ${ }^{6}$. Este método consiste en trazar una línea equidistante provisoria, que será ajustada, en un segundo tiempo, en función de circunstancias reconocidas como pertinentes, tal y como ciertos elementos geográficos. Por último, la Corte opera un examen negativo para verificar la ausencia de desproporción manifiesta, comparando los espacios marítimos atribuidos a ambas Partes con el largo de las costas consideradas para este efecto.

Sobre la base del derecho internacional aplicable, la Corte construyó el fallo por medio de un razonamiento en dos tiempos, partiendo por la cuestión de la existencia de un acuerdo internacional -argumento de Chile-, pues sólo en su defecto, se recurriría a la solución subsidiaria prevista por las normas internacionales -argumento de Perú-.

\section{Razonamiento en dos etapas de la Corte sobre la existencia y la extensión de la frontera convenida}

Sobre la base del derecho internacional consuetudinario, la Corte buscó primero si una frontera marítima había sido convenida por las Partes, concluyendo que éste había sido el caso, pero sólo hasta las 80 millas marinas (1). A partir de esa distancia, la Corte estableció una frontera de novo, aplicando la solución subsidiaria en derecho internacional al acuerdo de los Partes, es decir, la búsqueda de una solución sobre la base de la equidad (2).

\footnotetext{
Véase fallo, párr. 180. Sin embargo, como se trata sólo de un método y no de una norma, la equidistancia puede ser descartada según las circunstancias del caso. Esto fue efectivamente realizado por la CIJ en el diferendo Nicaragua contra Honduras precitado (ver n. 6), en el que la Corte explicó que, debido a las circunstancias geográficas, la equidad exigía descartar la equidistancia y aplicar el método de la bisectriz.
} 


\section{Existencia de una frontera convenida entre las Partes hasta las 80 millas marinas ${ }^{7}$}

Para pronunciarse sobre la existencia de una frontera convenida entre Perú y Chile, la Corte interpretó los instrumentos jurídicos invocados por las Partes, concluyendo que una frontera había sido convenida (A). Luego, la Corte abordó la cuestión de su extensión, concluyendo que ésta se extendía hasta las 80 millas marinas (B).

\subsection{Análisis de los instrumentos jurídicos concluyendo a la existencia de una frontera}

\subsubsection{Las proclamaciones unilaterales de Chile y Perú del año 1947}

La Corte comenzó su análisis con las proclamaciones unilaterales de las Partes de 1947, en las que tanto Chile como Perú revindicaban derechos marítimos hasta una distancia de 200 millas marinas. El decreto supremo n 781 adoptado por el Presidente de Perú el $1^{\circ}$ de agosto de 1947 toma en consideración la proclamación de Chile del 23 de junio del mismo año -entre otras-, y declara:

"[Perú] ejercerá dicho control y protección sobre el mar adyacente a las costas del territorio peruano en una zona comprendida entre esas costas y una línea imaginaria paralela a ellas y trazada sobre el mar a una distancia de doscientas (200) millas marinas, medida siguiendo la línea de los paralelos geográficos" ${ }^{\prime 8}$.

La Corte observó que las proclamaciones de las Partes intervinieron en un contexto donde varios Estados revindicaban nuevos derechos marítimos 9 . Sin embargo, siendo declaraciones unilaterales, la Corte descartó su importancia para decidir sobre la existencia de un acuerdo internacional.

\subsubsection{La Declaración de Santiago de 1952}

La Corte prosiguió con el estudio de los instrumentos concluidos por Perú, Chile y Ecuador en los años 1950 en el marco de la Conferencia sobre Explotación y Conservación de Recursos Marítimos del Pacífico Sur. La CIJ comenzó por la Declaración de Soberanía sobre la Zona Marítima de 200 Millas - "Declaración de Santiago"- del 18 de agosto de 1952 y observó que, pese a la apelación “declaración”, se trata de un tratado internacional (el derecho internacional, como lo hemos visto, no es

\footnotetext{
7 Punto 3 del dispositivo, adoptado con diez votos a favor (jueces Abraham, Bennouna, Cançado Trindade, Donoghue, Keith, Owada, Sepúlveda-Amor, Skotnikov, Yusuf y juez ad hoc Guillaume) y seis en contra (jueces Bhandari, Gaja, Sebutinde, Tomka, Xue y juez ad hoc Orrego Vicuña).

8 Perú, Presidente de la República, 1947, Decreto Supremo No 781, 1º de agosto de 1947, párr. 3.

9 Movimiento iniciado por la Proclamación del Presidente Truman de Estados Unidos, el 28 de septiembre de 1945.
} 
formalista). La Corte examinó el parágrafo IV de la Declaración, que, según Chile, establecía implícitamente la frontera marítima entre las Partes. El texto se lee como sigue:

"IV. En el caso de territorio insular, la zona de 200 millas marinas se aplicará en todo el contorno de la isla o grupo de islas.

Si una isla o grupo de islas pertenecientes a uno de los países declarantes estuviere a menos de 200 millas marinas de la zona marítima general que corresponde a otro de ellos, la zona marítima de esta isla o grupo de islas quedará limitada por el paralelo del punto en que llega al mar la frontera terrestre de los Estados respectivos".

Según Chile, esta disposición implicaba que la frontera de las zonas marítimas generadas por las costas continentales ya estaba definida por el paralelo geográfico, que permitiría, además, delimitar las zonas generadas por islas que se sobrepusieran a dichas zonas. Chile invocaba las Actas de los trabajos preparatorios de la Declaración, que establecen que el delegado de Ecuador expresó:

"[Q]ue convendría dar más claridad al artículo $3^{\circ}$ [parágrafo IV de la versión final], a fin de evitar cualquier error de interpretación de la zona de interferencia en el caso de islas y sugirió que la declaración se redactara sobre la base de que la línea limítrofe de la zona jurisdiccional de cada país fuera el paralelo respectivo desde el punto en que la frontera de los países toca o llega al mar" ${ }^{p 10}$.

Las Actas registran que "[t]odos los delegados estuvieron conformes con esta proposición"11.

La Corte interpretó estos instrumentos aplicando el artículo 31 de la Convención de Viena, relativo al sentido ordinario del texto según el contexto y el objetivo del tratado. La Corte observó que el parágrafo IV sólo tenía el objetivo específico de delimitar la frontera de las zonas generadas por las islas sin contener una disposición general ${ }^{12}$. El fallo afirma enseguida que, siendo el sentido del texto claro, no era necesario analizar los trabajos preparatorios que son métodos auxiliares conforme al artículo 32 de la Convención de Viena. Aun así, la Corte analizó la preocupación expresada por el delegado de Ecuador, concluyendo que ésta confirmaba el sentido ordinario del texto, pues tenía el mismo objetivo, es decir, clarificar la situación en presencia de islas ${ }^{13}$. La Corte descartó así la importancia de la Declaración de Santiago en el establecimiento de una frontera marítima entre las Partes.

\footnotetext{
10 Actas de la Primera Sesión de la Comisión de Asuntos Jurídicos de la Conferencia de 1952, 11 de agosto de 1952 a las 4 P.M., Anexo 56 de la Memoria, p. 2.

11 Idem.

12 Véase fallo, párr. 62.

13 Véase fallo, párr. 68.
} 
Sin embargo, la aplicación de las normas de interpretación de tratados podría haber dictado otra solución. Según la opinión común disidente de los jueces Bhandari, Gaja, Xue y del juez ad hoc nombrado por Chile Orrego Vicuña ${ }^{14}$-en adelante "opinión común disidente"-, la Corte debiese haber interpretado el parágrafo IV de la Declaración de Santiago conforme al principio del efecto útil, de tal manera que la disposición en cuestión tuviera los efectos jurídicos apropiados en el marco del tratado. Los jueces concluyen, tal y como argumentaba Chile, que el texto del parágrafo IV implicaba que la frontera generada por las costas continentales estaba también delimitada por el paralelo geográfico, solución que, según los jueces, fue confirmada por los acuerdos de 195415 .

\subsubsection{El Convenio Complementario a la Declaración de Santiago y el Convenio sobre Zona Especial} Fronteriza Maritima de 1954

Luego de descartar la importancia de la Declaración de Santiago, la Corte se interesó a tres de los seis acuerdos concluidos en 1954. Todos los acuerdos concluidos en 1954 contienen la siguiente cláusula tipo:

"Todo lo establecido en el presente Convenio se entenderá ser parte integrante, complementaria y que no deroga las resoluciones y acuerdos adoptados en la Conferencia sobre Explotación y Conservación de las Riquezas Marítimas del Pacífico Sur, celebrada en Santiago de Chile, en agosto de 1952"16.

La Corte sólo examinó con atención el Convenio sobre Zona Especial Fronteriza Marítima -en adelante "Convenio sobre Zona Especial"-, descartando la importancia, en nuestra opinión fundamental, del Convenio Complementario a la Declaración de Santiago -en adelante "Convenio Complementario".

\subsubsection{Convenio Complementario a la Declaración de Soberanía sobre la Zona Marítima de 200 Millas}

Chile argumentaba que el Convenio Complementario reafirmaba la frontera convenida implícitamente en 1952. Para esto, Chile invocaba las Actas de los trabajos preparatorios del Convenio, que establecen que el delegado de Ecuador expresó la necesidad de integrar:

14 El artículo 31, párrs. 2 y 3 del estatuto de la CIJ prevé la posibilidad de cada Parte a un diferendo de nombrar a un juez -juez ad hoc- en caso de que ningún juez de la Corte tenga la nacionalidad de dicha Parte. En virtud de esta disposición y conforme a los artículos 35 a 37 del reglamento de la CIJ, Chile nombró a Francisco Orrego Vicuña y Perú a Gilbert Guillaume, ex presidente de la Corte.

15 Véase opinión común disidente, párr. 35.

16 Véase Memoria, p. 84 y fallo, párr. 73. 
" $[\mathrm{U}] \mathrm{n}$ artículo complementario que aclare el concepto de la línea divisoria del mar jurisdiccional que ya ha sido expuesto en la Conferencia de Santiago, pero que no está demás repetir aquî" ${ }^{\prime 17}$.

Las Actas establecen que los delegados de Chile y Perú estimaron que no era necesario introducir un nuevo artículo con este fin, bastando introducir en las Actas:

"[Q]ue los tres países consideran resuelto el punto de la línea divisoria de las aguas jurisdiccionales, que es el paralelo que parte del punto en que la frontera terrestre de ambos países establecida en la Conferencia"18.

Las Actas establecen que el delegado peruano precisó:

"[E]sta conformidad ya quedó establecida en la Conferencia de Santiago como consta en el acta respectiva a pedido del Delegado del Ecuador" ${ }^{\prime 19}$.

Sin embargo, la Corte, habiendo descartado previamente la importancia de la Declaración de Santiago para el acuerdo sobre la frontera marítima, desestimó con ella la relevancia del Convenio Complementario ${ }^{20}$. De manera inexplicable, los trabajos preparatorios de 1954 ni siquiera fueron reproducidos en el fallo, aún sabiendo que las Actas establecen expresamente el sentido que las partes pretendían dar a la Declaración de Santiago y la razón por la que no habían convenido de manera explícita la delimitación marítima. Si bien es cierto que el artículo 32 de la Convención de Viena prevé el recurso a los trabajos preparatorios sólo como método complementario de interpretación, la aplicación de las normas debe adaptarse a las circunstancias del caso. En el diferendo entre Chile y Perú, los trabajos preparatorios de 1954 tenían una importancia fundamental para determinar el sentido tanto de la Declaración de 1952 como de los acuerdos posteriores.

Sólo algunas declaraciones y opiniones individuales adjuntas al fallo informan sobre la existencia de los trabajos preparatorios de 1954 y proveen un análisis jurídico al respecto $^{21}$. En la declaración del juez Tomka -que constituye más bien una opinión disidente- el presidente de la Corte observa que los trabajos preparatorios del Convenio Complementario establecen de manera clara que las Partes ya habían resuelto la cuestión de las fronteras marítimas generadas por las costas continentales incluso antes de concluir la Declaración de Santiago 22.

17 Actas de la Primera Sesión de la Comisión I de la Conferencia Interestatal de 1954, 2 de diciembre de 1954 a las 10.00 A.M., Anexo 38 de la Contramemoria, p. 3.

18 Ibidem.

19 Idem, p. 4.

20 Véase fallo, párr. 77.

21 Véase declaración ajunta del juez Tomka (párrs. 14-15) y opinión común disidente (párr. 23).

22 Véase declaración ajunta del juez Tomka, párr. 22. 


\subsubsection{Convenio sobre Zona Especial Fronteriza Maritima}

Luego de descartar rápidamente el Convenio Complementario, el fallo se concentró en el Convenio sobre Zona Especial del 4 de diciembre de 1954, que prevé:

"Considerando que la experiencia ha demostrado que debido a las dificultades que encuentran las embarcaciones de poco porte tripuladas por gente de mar con escasos conocimientos de náutica o que carecen de los instrumentos necesarios para determinar con exactitud su posición en alta mar, se producen con frecuencia, de modo inocente y accidental, violaciones de la frontera marítima entre los Estados vecinos [...]

Establécese una Zona Especial, a partir de las 12 millas marinas de la costa, de 10 millas marinas de ancho a cada lado del paralelo que constituye el límite marítimo entre los dos países".

Tras observar que las Partes no habían distinguido entre las expresiones "frontera" y "límite", y que por ende la Corte tampoco debía hacerlo, el fallo afirma:

"[L]a formulación del acuerdo de 1954 relativo a una zona especial fronteriza marítima, en particular del artículo primero, interpretado a la luz del preámbulo, es clara: reconoce, en el marco de un acuerdo internacional vinculante, que una frontera marítima ya existe" $^{\text {23 }}$.

Para la Corte, la frontera no fue establecida por el Convenio sobre Zona Especial, sino que éste sólo se limita a constatarlo. La frontera había sido convenida tácitamente en el periodo comprendido entre 1952 y 1954.

Sin embargo, como indica el juez Sepúlveda-Amor en su declaración adjunta -que constituye una verdadera opinión disidente, pese a que el juez votó con la mayoría en todo el dispositivo- nada ocurrió entre 1952 y 1954 que justificase la existencia de un acuerdo tácito ${ }^{24}$, por lo que tal acuerdo no existe ${ }^{25}$. En efecto, si se desestima el rol fundamental de la Declaración de Santiago y de los trabajos preparatorios de 1954, sólo se puede constatar lo mismo que el juez Sepúlveda-Amor. No obstante, y es aquí donde la argumentación del juez no encuentra sustento -y que el análisis del fallo se desvanece-, dicho acuerdo -inexistente para Sepúlveda-Amor y confusamente tácito para el resto de la mayoría- sirvió de base para el Convenio sobre Zona Especial, que habla de "violación de fronteras".

La posición difícilmente justificable del fallo y la contradicción evidente entre el razonamiento del juez Sepúlveda-Amor y el acuerdo expreso de las Partes en 1954 no eran, sin embargo, necesarias. Como hemos visto, una aplicación adaptada al caso de las

23 Véase fallo, párr. 90.

24 Véase declaración adjunta del juez Sepúlveda-Amor, párrs. 8-9.

25 Idem, párr. 16. 
normas de interpretación de tratados hubiese permitido sostener la existencia de una frontera convenida -que motivó la conclusión del Convenio sobre Zona Especial- sobre fundamentos jurídicos sólidos. La ausencia de dichos fundamentos en la constatación del acuerdo tácito, explica las dificultades posteriores encontradas por la Corte para determinar la extensión de la frontera así convenida.

\subsection{Extensión de la frontera convenida hasta las 80 millas marinas}

Una vez constatada la existencia de una frontera convenida por las Partes la Corte debía resolver la cuestión de su extensión, puesto que el acuerdo, siendo tácito, no podía prever nada en este sentido y que la Corte había descartado los instrumentos que consideraban la extensión hasta las 200 millas marinas - especialmente las proclamaciones unilaterales de 1947 y la Declaración de Santiago -. Para interpretar la extensión prevista por el acuerdo tácito, la Corte analizó la práctica de las Partes en dicha época, concentrándose en las actividades pesqueras y en el estado del derecho internacional del mar, que no reconocía todavía derechos marítimos de los Estados costeros hasta una distancia de 200 millas marinas.

Sobre esta base, la Corte concluye:

"Considerando las actividades pesqueras de las Partes en ese periodo, que se ejercían hasta una distancia de aproximadamente 60 millas marinas a partir de los principales puertos de la región, así como también la práctica de los otros Estados y los trabajos de la Comisión de Derecho Internacional en materia de derecho marítimo, la Corte estima que los elementos disponibles son insuficientes para permitirle concluir que la frontera marítima convenida, que seguía el paralelo, se extendía mas allá de 80 millas marinas desde su punto inicial" ${ }^{\prime 26}$.

El razonamiento seguido por la Corte sorprende en varios aspectos. En primer lugar, el fallo no sustenta positivamente la extensión de la frontera convenida. Como indica la jueza Sebutinde en su opinión disidente, el hecho de que la frontera convenida no pudiese en ningún caso extenderse más allá de 80 millas marinas no implica que ésta deba extenderse hasta esa distancia ${ }^{27}$. Por otro lado, como observa el juez ad hoc Orrego Vicuña en su opinión individual, reducir la extensión de la frontera a las actividades pesqueras de la época equivale a reducir el acuerdo tácito sobre la frontera marítima al objetivo específico del Convenio sobre Zona Especial, pese a que éste, según la Corte, no establece la frontera sino que sólo la constata ${ }^{28}$.

26 Véase fallo, párr. 117.

Véase opinión disidente de la jueza Sebutinde, párr. 14.

28 Véase opinión individual del juez ad hoc Orrego Vicuña, párr. 16. 
Es más, si uno de los fundamentos del fallo para rechazar el acuerdo hasta las 200 millas marinas es el estado del derecho marítimo internacional -como lo plantea el juez ad hoc Orrego Vicuña ${ }^{29}$, entonces nada explica que la frontera se extienda más allá de la distancia reconocida en aquella época por el derecho internacional reflejado en las convenciones de Ginebra: 12 millas marinas ${ }^{30}$. No obstante, como observa el juez Tomka ${ }^{31}$ y la opinión común disidente ${ }^{32}$, el hecho de que en ese momento la frontera marítima de 200 millas marinas no fuera conforme al derecho internacional general, sólo implica que la frontera así convenida no podía hacerse valer jurídicamente respecto de terceros Estados, pero no que ésta no sería válida entre las Partes en miras de la evolución del derecho marítimo internacional.

\section{Trazado de la frontera de novo sobre la base de la equidad a partir de las 80 millas marinas $^{33}$}

Una vez establecida la extensión de la frontera marítima convenida, la Corte utilizó el método en tres etapas consagrado en su jurisprudencia para buscar una solución sobre la base de la equidad en la distancia restante. Así, la Corte trazó una línea equidistante provisoria para dividir las zonas marítimas de ambas partes ${ }^{34}$, indicando luego que no habían circunstancias pertinentes que justificaran su ajuste ${ }^{35}$. Finalmente, invocando la dificultad de hacer el control de la desproporción manifiesta debido al establecimiento convencional de un gran segmento de la frontera, la Corte indicó que no iba a realizar dicho examen final ${ }^{36}$. La frontera marítima a partir de las 80 millas marinas siguió de este modo la línea equidistante inicial.

Idem, párr. 22.

30 Véase artículo 24, párr. 2, de la Convención de Ginebra sobre el Mar territorial y la Zona Contigua, adoptada el 29 de abril de 1958.

31 Véase declaración adjunta del juez Tomka, párr. 6.

32 Véase opinión común disidente, párr. 17.

33 Punto 4 del dispositivo, adoptado con la misma mayoría que el punto 3 (ver n. 8).

34 Véase fallo, párr. 184.

Idem, párr. 191.

Idem, párrs. 193-194. 


\section{La vocación general de la frontera y su inicio en el hito $\mathbf{N}^{\mathbf{o}} 1^{37}$}

Para definir la vocación de la frontera -si una frontera única delimita todos los espacios marítimos (los fondos marinos, el subsuelo y las aguas que los cubren)- la Corte recurrió a las proclamaciones unilaterales de 1947 y a la Declaración de Santiago de 1952, afirmando que ninguno de estos actos distinguía según las distintas zonas y que debía entenderse, entonces, que las Partes pretendían establecer una frontera marítima única de vocación general ${ }^{38}$. El recurso de la Corte a estos instrumentos sorprende, pues nada explica que estos instrumentos sean tomados en cuenta a este estado del análisis siendo que fueron descartados para definir tanto la existencia como la extensión de la frontera convenida por las Partes, como bien observa el juez Skotnikov, quien votó sin embargo con la mayoría en todo el dispositivo- en su declaración adjunta ${ }^{39}$.

Siguiendo, en cambio, la lógica general del fallo, la Corte determinó el punto inicial de la frontera marítima a partir de la práctica subsecuente de las Partes, sin referirse al parágrafo IV de la Declaración de Santiago que la establece a partir "[d]el paralelo del punto en que llega al mar la frontera terrestre". Por lo mismo, la Corte no se interesó al punto inicial de la frontera terrestre, establecido en el denominado "Punto de la Concordia" por el Tratado de Lima concluido por Perú y Chile el 3 de junio de 192940, afirmando de manera expresa que este punto pudiese no coincidir con el punto inicial de la frontera marítima ${ }^{41}$. Para la Corte, en cambio, fueron centrales los acuerdos entre Chile y Perú de los años 1968 y 1969 relativos a la instalación de faros para materializar la frontera marítima, que tomaban como referente el paralelo que pasa por el hito n. ${ }^{\circ} 1^{42}$.

He aquí, no obstante, una contradicción tanto del argumento chileno como del peruano, pues si se afirma, como hacía Chile, que la frontera fue implícitamente reconocida por la Declaración de Santiago, entonces el punto inicial debiera ser aquél que este instrumento señala. Inversamente, para invocar el Punto de la Concordia como inicio de la frontera marítima, como afirmaba Perú, se debe reconocer la importancia fundamental de la Declaración de Santiago en la delimitación, pues en el caso contrario, nada liga el inicio de la frontera marítima al inicio de la frontera terrestre.

Como hemos visto, el Tratado de Lima establece el inicio de la frontera terrestre en el "Punto de la Concordia", la materialización siendo dejada a una comisión mixta cuyo trabajo se extendió entre 1929 y 1930. El mandato de la Comisión -establecido de forma

37 Punto 1 del dispositivo, adoptado con quince votos a favor y uno en contra (juez Gaja).

38 Véase fallo, párr. 102.

39 Véase declaración adjunta del juez Skotnikov, párr. 3.

40 Véase el artículo 2 inciso 1 del Tratado de Lima.

41 Véase fallo, párr. 175.

42 Ibidem, párr. 174-175. 
idéntica por los dos Estados- reconocía el inicio de la frontera en el "punto de intersección del arco trazado con la orilla del mar"43, exigiendo la instalación de un hito "lo más próximo al mar posible, donde quede a cubierto de ser destruido por las aguas del océano" 44 . Así, el mandato reconoce que el punto inicial de la frontera no es aquél que será materializado por el hito fronterizo. Siguiendo al juez Gaja, sólo podría considerarse que el paralelo que pasa por el hito $\mathrm{n}^{\circ}{ }^{\circ} 1$ es el punto inicial de la frontera marítima si se logra probar que un acuerdo posterior de las Partes modificó este punto, disociándolo del inicio de la frontera terrestre. No obstante, parece extremadamente dudoso que los acuerdos de 1968-1969, que tenían solamente el objetivo práctico de permitir a los barcos pequeños reconocer fácilmente la frontera marítima, tuvieran el fin general de modificar la delimitación ${ }^{45}$.

Entonces, la aceptación por parte de la Corte del punto inicial de la frontera marítima invocado por Chile -el hito n. ${ }^{\circ} 1$ - tiene directa relación con el descarte previo de la Declaración de 1952 para determinar la existencia de la frontera. Así como por las actividades pesqueras respecto de la extensión de la frontera convenida, la Corte debía encontrar algo que la informara sobre el inicio de la frontera y esto fueron los acuerdos de 1968-1969. Sin embargo, si consideramos que la Declaración de 1952 sí tuvo una importancia fundamental en el reconocimiento de la frontera marítima, entonces la misma importancia debe darse al punto inicial fijado por dicho acuerdo y por lo mismo, al punto convenido en el Tratado de Lima.

Cabe destacar que la Corte no podía pronunciarse sobre la delimitación de la frontera terrestre, puesto que el artículo VI del Pacto de Bogotá, fundamento de su competencia, excluye las cuestiones resueltas por medio de tratados anteriores al Pacto, lo que incluye al Tratado de Lima. Sin embargo, como observa el juez Gaja, esto no impedía a la Corte referirse al Tratado de Lima, pero sólo con el fin de establecer la frontera marítima y no para operar la delimitación de la frontera terrestre ${ }^{46}$. Así, cualquier conclusión de la Corte respecto de la frontera terrestre per se no hubiese tenido valor vinculante para las Partes, pues la Corte no tenía competencia ratione materiae en esta área.

43 Véase Memoria, pp. 124-125.

44 Idem.

45 Véase declaración adjunta del juez Gaja, párr. 4.

46 Idem, párr. 2. 


\section{Conclusión}

La proliferación de posiciones individuales de los jueces de la Corte, ya sea por medio de declaraciones adjuntas, de opiniones concurrentes o disidentes, pone en evidencia la debilidad del sustento jurídico del fallo. Incluso dentro de la mayoría que apoyó los puntos más controversiales del dispositivo - la existencia de un acuerdo tácito y la extensión de la frontera así convenida- se observan diferencias irreconciliables en el fundamento de la posición de cada juez ${ }^{47}$. En este sentido, la elaboración por parte del juez Tomka, presidente de la Corte, y del juez Sepúlveda-Amor, vice-presidente, de simples declaraciones con contenidos, sin embargo, propios de opiniones disidentes, pudiese indicar la voluntad de conservar la autoridad de un fallo que desintegró la unidad de la Corte, en un contexto regional extremamente delicado en materia de delimitación judicial de fronteras ${ }^{48}$.

Las dificultades en las cuestiones de forma, sin embargo, no hacen sino traducir aquéllas de fondo en el razonamiento de la Corte, que sólo pueden explicarse por la voluntad de adoptar una solución de compromiso ${ }^{49}$. En efecto, si la Corte hubiese reconocido el valor jurídico de la Declaración de Santiago respecto de la delimitación de la frontera apoyándose, particularmente, en los trabajos preparatorios del Convenio Complementario de 1954-, entonces hubiese sido imposible adoptar una solución que satisficiese en parte las pretensiones de Perú, pues la Declaración reconocía una extensión de 200 millas marinas. Inversamente, si se afirmaba que no existía acuerdo alguno -lo que hubiese, además, sido contrario al texto mismo del Convenio sobre Zona Especial-, entonces la frontera hubiese sido delimitada completamente de novo, excluyendo la totalidad de las pretensiones chilenas. Pareciera así que, para evitar una solución de "todo o nada", la Corte descartó el valor jurídico determinante de la Declaración de Santiago y se esmeró en encontrar un fundamento distinto, en realidad inexistente, para reconocer una frontera convenida por las Partes hasta una distancia menor a 200 millas marítimas. Pero una vez descartada la Declaración de Santiago, y con ella los trabajos preparatorios del Convenio Complementario, el fundamento era difícil, sino imposible, de encontrar. Prueba de ello es el hecho de que las circunstancias del acuerdo permanecen totalmente obscuras en el fallo. La existencia de un acuerdo tácito, por lo demás, nunca fue argumentada por las Partes y, si bien no cabe duda de que esto no impide a la Corte considerar un punto

47 Basta evocar la declaraciones del juez Sepúlveda-Amor, que no reconoce la existencia de un acuerdo tácito y del juez Skotnikov, que plantea que la Corte debiese haber considerado las proclamaciones de 1947 y la Declaración de Santiago para determinar la existencia y la extensión de la frontera convenida.

48 Véase, en este sentido, BoEglin, N., "La Cour Internationale de Justice, le Pérou, le Chili et quelques questions de forme" [en línea]. Sentinelle, $\mathrm{n}^{\circ} 377,9$ de febrero de 2014,. Disponible en: <http://www.sentinelle-droitinternational.fr/bulletins/a2014/20140209_bull_377/bulletin_sentinelle_377.php\#701> [consulta: 12 de febrero de 2014].

49 Véase, en este sentido, la opinión individual del juez ad hoc Orrego Vicuña, párr. 27. 
incluso como parte fundamental de la decisión, parece dudoso que, tratándose de un acuerdo internacional que supuestamente refleja la intención de ambos Estados, la existencia de tal acuerdo no haya sido nunca discutida por las Partes en un proceso judicial que duró más de seis años ${ }^{50}$.

En definitiva, las consideraciones sobre las que se basa el fallo se acercan más a la búsqueda de una solución sobre la base de la equidad que a la búsqueda de la existencia de un acuerdo entre las Partes. La Corte puede y debe, conforme al derecho internacional consuetudinario, recurrir a la equidad para delimitar los espacios marítimos en la ZEE y la plataforma continental, pero sólo cuando las Partes no han convenido previamente una frontera. El problema fundamental, aquí, es la voluntad implícita de la Corte de invertir el orden jerárquico de las normas y de recurrir, de manera global, a la equidad para inspirar la decisión cuando la equidad no es sino una solución subsidiaria para la delimitación marítima en derecho internacional.

50 La jueza Donoghue, que se unió a la mayoría en todo el dispositivo, reconoce en su declaración adjunta que la Corte debiese haber utilizado mecanismos procedurales - como pedir a las Partes argumentaciones adicionales o emitir un fallo parcial - "that may offer advantages when issues that are important to the Court's conclusions have not been squarely addressed by the parties [...] By making use of procedural approaches such as those noted here, the Court could further enrich its practice and jurisprudence". 